\title{
Wenn Antibiotika nicht mehr helfen
}

DOI: $10.1007 / \mathrm{s} 12268-014-0449-7$

(C) Springer-Verlag 2014

- Heutzutage sterben in Europa trotz bester medizinischer Versorgung pro Jahr mehr als 25.000 Menschen an Krankenhausinfektionen mit resistenten Bakterien. Die Weltgesundheitsorganisation WHO fasst in ihrem aktuellen Bericht ${ }^{1}$ erstmals global die alarmierende Situation der Antibiotikaresistenz zusammen und warnt dabei ausdrücklich vor einer bevorstehenden postantibiotischen Ära, in der gewöhnliche Infektionen wieder tödlich sein können. Als vorherrschende Problemkeime gelten die ESKAPE-Erreger (Enterococcus faecium, Staphylococcus aureus, Klebsiella pneumoniae, Acinetobacter baumannii, Pseudomonas aeruginosa und Enterobacter spec.). So versagen derzeit Antibiotika der letzten Instanz, wie Cephalosporine der dritten Generation, Carbapeneme oder Fluorochinolone, bei der Therapie von Infektionen mit den meist multiresistenten Gram-negativen Keimen. Bei den Gram-positiven Erregern sind insbesondere die weltweiten Zahlen der MRSA-Infektionen (Methicillin-resistenter Staphylococcus aureus) besorgniserregend, mit über 25 Prozent in Europa und bis zu 90 Prozent in den erfassten Gebieten Afrikas.

Die rasant zunehmende Resistenzverbreitung wird im Wesentlichen dem sorglosen Umgang mit Antibiotika in der Human- und Tiermedizin sowie mangelnder Krankenhaushygiene zugeschrieben. Ein sparsamer und vor allem gezielter Gebrauch von Antibiotika sowie eine ausreichende Therapiezeit sind die wichtigsten Gegenmaßnahmen. Um Krankheitskeime zukünftig schneller zu identifizieren und sie dann gezielt zu bekämpfen, werden dringend neue Diagnostikmethoden benötigt. Sie ermöglichen dann den gezielten Einsatz von Schmalspektrum-Antibiotika.

\footnotetext{
WHO (2014) Antimicrobial resistance: global report on surveillance, www.who.int/drugresistance/docu ments/surveillancereport
}

Zusätzlich stellt die Förderung internationaler Netzwerke wie CAESAR (Central Asian and Eastern European Surveillance of Antimicrobial Resistance) oder TATFAR (Transatlantic Taskforce on Antimicrobial Resistance) eine wichtige Maßnahme dar, um Informationen über regional aufkommende Resistenzen effizienter und koordiniert zu sammeln und darauf zu reagieren.

Der kontinuierlich steigenden bakteriellen Resistenzentwicklung steht allerdings die stetige Abnahme der Entwicklung neuer Antibiotika entgegen. Lediglich vier Substanzen der letzten 40 Jahre basieren auf neuen Antibiotikaklassen, trotz zahlreicher moderner Hochdurchsatzscreenings. Von vielen Fehlschlägen entmutigt, haben zahlreiche große Pharmaunternehmen in jüngerer Vergangenheit ihre antibiotische Wirkstoffforschung eingestellt. Hinzu kommt, dass bei den niedrigen Preisen für lebensrettende Antibiotika die Entwicklung von Therapeutika zur Behandlung chronischer Erkrankungen wesentlich rentabler erscheint.

Um heute auf der Suche nach Antibiotika mit neuartigen Wirkmechanismen einen entscheidenden Durchbruch zu erlangen, ist es dringend nötig, alle verfügbaren Ressourcen in einem integrierten Prozess der antibiotischen Leitstrukturfindung und -optimierung zu bündeln. Auf stofflicher Ebene umfasst dies sowohl die Quelle der niedermolekularen Wirkstoffpools als auch Naturstoffbanken, auf methodischer Ebene die Techniken der funktionellen Genomanalyse, die Möglichkeiten der kombinatorischen Chemie zusammen mit klassischen medizinal-chemischen Syntheseverfahren, unterstützt durch Strukturinformationen und molekulare Modellierung. Unerlässlich ist zudem eine verstärkte Grundlagenforschung, um ein tieferes Verständnis der molekularen Wirkmechanismen der Substanzen sowie der Antibiotikawirkung auf zellulärer Ebene zu erlangen.

Mit den genannten verfügbaren experimentellen Techniken und dem Pool an eta- bliertem biochemischem, mikro- und molekularbiologischem Wissen ist die moderne Antibiotikaforschung heute prinzipiell in einer guten Ausgangsposition. Es müssen jedoch neue Finanzierungsmodelle für transnationale Forschung und Entwicklung hinzukommen und die Zulassungsverfahren für neue Antibiotika erleichtert und beschleunigt werden, damit die Entwicklung neuer Antibiotika wieder attraktiv wird und die Pharmaindustrie weltweit wieder intensiv in die Antibiotikaforschung einsteigt.

Die Innovative Medicines-Initiative (IMI) der Europäischen Union mit Mitgliedern der European Federation of Pharmaceutical Industries and Associations (EFPIA) fördert und finanziert gemeinsame Forschungsprojekte von Industrie und Hochschulen. In diesem Konsortium werden aktuell auch Projekte der Antibiotika-Entwicklung bearbeitet. Dies ist ein erster wichtiger Schritt zur Generierung der dringend benötigten Resistenz-brechenden Antibiotika in Europa. Eine Forschungsförderung, die auch die späten klinischen Phasen unterstützt, wäre auch national äußerst wünschenswert.

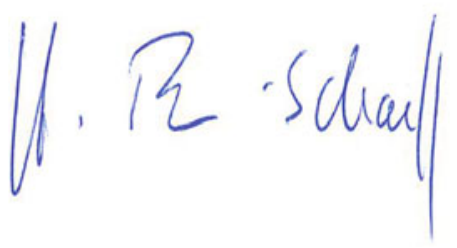

Helga Rübsamen-Schaeff, CEO, AiCuris GmbH \& Co. KG, Wuppertal unter Mitarbeit von Daniela Münch und Dr. Cuong Vuong

Korrespondenzadresse:

Prof. Dr. Helga Rübsamen-Schaeff

AiCuris $\mathrm{GmbH} \&$ Co. KG

Friedrich-Ebert-Straße 475

D-42117 Wuppertal

Tel.: 0202-31763-0

Fax: 0202-31763-1601

info@aicuris.com 